\title{
Enantioselective Total Synthesis of Semperoside A
}

\author{
Paolo Piccinini, Giovanni Vidari,* and Giuseppe Zanoni \\ Dipartimento di Chimica Organica, Università di Pavia, \\ Viale Taramelli,10, 27100 Pavia - Italy
}

\section{Supporting Information}

General Experimental Procedure: For a general experimental procedure of preparation of acetate 9 see previous papers. ${ }^{1}$

\section{[4aS,5Z,7S,7aR]-7-Methyl-4,4a,7,7a-tetrahydro-1H-cyclopenta[c]pyran-3-one (10).}

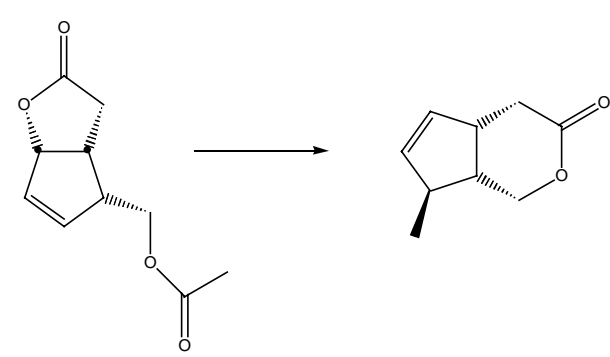

$\mathrm{MeMgBr}\left(3 \mathrm{M}\right.$ in $\left.\mathrm{Et}_{2} \mathrm{O}, 2.5 \mathrm{~mL}\right)$ was added dropwise to a stirred solution of $\mathrm{CuBrMe}_{2} \mathrm{~S}(1.55 \mathrm{~g}, 7.5 \mathrm{mmol})$ in dry THF $(15 \mathrm{~mL})$ and $\mathrm{Me}_{2} \mathrm{~S}(8.2 \mathrm{~mL})$ at $-20^{\circ} \mathrm{C}$ (cryostat). The yellow cuprate suspension was kept at $-20^{\circ} \mathrm{C}$ for $10 \mathrm{~min}$, then cooled to $-45^{\circ} \mathrm{C}$, and acetate (-)-9 (500 $\mathrm{mg}, 2.5 \mathrm{mmol}$ ), in dry THF was slowly added by syringe. After $3 \mathrm{~h}$ the mixture was warmed to room temperature, poured onto a $1 \mathrm{~N} \mathrm{NaOH}$ solution, and stirring was continued for an additional $1 \mathrm{~h}$. The solution was acidified to $\mathrm{pH}=2$ with $1 \mathrm{~N}$ $\mathrm{HCl}$ and then extracted with $\mathrm{Et}_{2} \mathrm{O}$. The organic layer was concentrated to about $15 \mathrm{~mL}$; a catalytic amount of $p$-TsOH was added and the solution was stirred until complete lactonisation $(8 \mathrm{~h})$. The solution was diluted with $\mathrm{CH}_{2} \mathrm{Cl}_{2}$ and washed with $5 \%$ aq. $\mathrm{NaHCO}_{3}$. The aqueous layer was extracted with $\mathrm{CH}_{2} \mathrm{Cl}_{2}$. The combined organic layers were dried over $\mathrm{MgSO}_{4}$ and solvent was removed. Separation of the residue by column chromatography (10 g of silica gel, hexane / EtOAc $=8: 2)$ gave lactone $10(317 \mathrm{mg}, 2.1 \mathrm{mmol}$, yield $=85 \%)$; m.p.: $77^{\circ}-78^{\circ} \mathrm{C} .[\alpha]_{\mathrm{D}}=+191.3(c=1.3$, $\mathrm{CH}_{2} \mathrm{Cl}_{2}$ ); IR (nujol): 3047, 2925, 1728, 1461, 1238, $1065 \mathrm{~cm}^{-1} ;{ }^{1} \mathrm{H}-\mathrm{NMR}\left(300 \mathrm{MHz}, \mathrm{CDCl}_{3}\right) \delta 5.70$ $(\mathrm{dt}, J=5.5,2.0 \mathrm{~Hz}, 1 \mathrm{H}), 5.52(\mathrm{dt}, J=5.5,2.0 \mathrm{~Hz}, 1 \mathrm{H}), 4.32(\mathrm{dd}, J=4.5,11.5 \mathrm{~Hz}, 1 \mathrm{H}), 4.08(\mathrm{dd}, J=$ $6.5,11.5 \mathrm{~Hz}, 1 \mathrm{H}), 3.33-3.45(\mathrm{~m}, 1 \mathrm{H}), 2.68(\mathrm{dd}, J=7.0,15.0 \mathrm{~Hz}, 1 \mathrm{H}), 2.55-2.75(\mathrm{~m}, 1 \mathrm{H}), 2.34(\mathrm{dd}, J$ $=6.0,15.0 \mathrm{~Hz}, 1 \mathrm{H}), 2.20-2.28(\mathrm{~m}, 1 \mathrm{H}), 1.11(\mathrm{~d}, J=7.0 \mathrm{~Hz}, 3 \mathrm{H}) ;{ }^{13} \mathrm{C}-\mathrm{NMR}\left(75.2 \mathrm{MHz}, \mathrm{CDCl}_{3}\right) \delta$ $173.3(\mathrm{CO}), 137.1(\mathrm{CH}), 130.6(\mathrm{CH}), 69.5\left(\mathrm{CH}_{2}\right), 43.7(\mathrm{CH}), 42.9(\mathrm{CH}), 41.5(\mathrm{CH}), 33.7\left(\mathrm{CH}_{2}\right)$, $21.2\left(\mathrm{CH}_{3}\right)$. Anal. Calcd for $\mathrm{C}_{9} \mathrm{H}_{12} \mathrm{O}_{2}$ : C, 71.03; H, 7.95. Found: $\mathrm{C}, 71.14 ; \mathrm{H}, 7.87$.

\section{$[3 a R, 4 S, 5 R, 6 R, 6 a R]-4-H y d r o x y m e t h y l-6-i o d o-5-m e t h y l-h e x a h y d r o-c y c l o p e n t a[b]$ furan-2-one} (11).

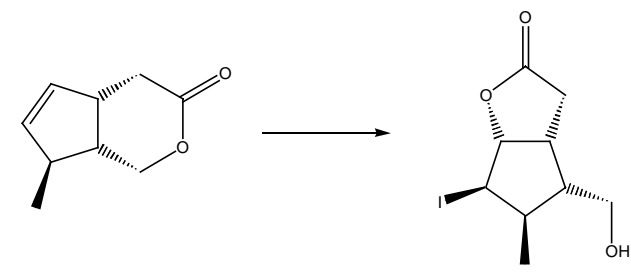

Lactone 10 (280 mg, $1.8 \mathrm{mmol})$ was added to a hydroalcoholic solution $\left(\mathrm{H}_{2} \mathrm{O}-\mathrm{EtOH} \mathrm{1:1}\right.$ ) of $\mathrm{NaOH}(72 \mathrm{mg}, 1.8$ $\mathrm{mmol})$ and the mixture was refluxed overnight, monitoring the reaction by TLC (hexane/EtOAc $=3: 7$ ). Solvent was evaporated under reduced pressure by aiding with addition of $\mathrm{CH}_{3} \mathrm{CN}$. The residue was washed with $\mathrm{Et}_{2} \mathrm{O}$ to remove unreacted olefin and the suspension was filtered. The recovered sodium salt was dissolved in $\mathrm{H}_{2} \mathrm{O}(20 \mathrm{~mL})$ and acidified to $\mathrm{pH}=7$ by the addition of dry ice. The neutralized solution was then added portionwise to a stirred solution of KI (996 mg, 6

\footnotetext{
${ }^{1}$ G. Zanoni; A. Porta; A. Meriggi; M. Franzini; G. Vidari J.Org.Chem., 2002, 67, 60-64

G. Zanoni; F. Agnelli; A. Meriggi; G. Vidari Tetrahedron Asimmetry, 2001, 12, 1779
} 
mmol $)$ and $\mathrm{I}_{2}(1.47 \mathrm{~g}, 5.8 \mathrm{mmol})$ in $\mathrm{H}_{2} \mathrm{O}(100 \mathrm{~mL})$ at $0^{\circ} \mathrm{C}$. The resulting mixture was stirred overnight. Solid $\mathrm{Na}_{2} \mathrm{SO}_{3}$ was added to destroy excess iodine. Solvent was evaporated and the residue was partitioned between $\mathrm{CH}_{2} \mathrm{Cl}_{2}$ and $\mathrm{H}_{2} \mathrm{O}$. The aqueous layer was reextracted with $\mathrm{CH}_{2} \mathrm{Cl}_{2}$. The combined organic layers were dried over $\mathrm{MgSO}_{4}$ and evaporated to give iodide 11 as nice colorless crystals $(504 \mathrm{mg}, 1.71 \mathrm{mmol}$, yield $=95 \%)$. $[\alpha]_{\mathrm{D}}=+38.02\left(c=1.075, \mathrm{CH}_{2} \mathrm{Cl}_{2}\right)$; IR (nujol) 3260, 2929, 1784, 1460, 1376, 1166, 1039, 1020, 995, $886 \mathrm{~cm}^{-1} ;{ }^{1} \mathrm{H}$ NMR (300 MHz, CDCl $\left.{ }_{3}\right) \delta 5.30$ $(\mathrm{d}, J=6 \mathrm{~Hz}, 1 \mathrm{H}), 4.60(\mathrm{~d}, J=4.3 \mathrm{~Hz}, 1 \mathrm{H}), 3.71(\mathrm{dd}, J=5.7,11 \mathrm{~Hz}, 1 \mathrm{H}), 3.88(\mathrm{dd}, J=6.9,11 \mathrm{~Hz}$, $1 \mathrm{H}), 3.28-3.4(\mathrm{~m}, 1 \mathrm{H}), 2.85(\mathrm{dd}, J=2,18.6 \mathrm{~Hz}, 1 \mathrm{H}), 2.70(\mathrm{dd}, J=10,18.6 \mathrm{~Hz}, 1 \mathrm{H}), 2.15-2.3(\mathrm{~m}$, $1 \mathrm{H}), 1.24-1.38(\mathrm{~m}, 1 \mathrm{H}), 1.08(\mathrm{~d}, J=6.2 \mathrm{~Hz}, 3 \mathrm{H}) ;{ }^{13} \mathrm{C}$ NMR $\left(75.2 \mathrm{MHz}, \mathrm{CDCl}_{3}\right) \delta 177.1(\mathrm{C}=\mathrm{O})$, $90.9(\mathrm{CH}), 59.8\left(\mathrm{CH}_{2}\right), 47.0(\mathrm{CH}), 42.7(\mathrm{CH}), 38.5(\mathrm{CH}), 36.9(\mathrm{CH}), 30.4\left(\mathrm{CH}_{2}\right), 19.2\left(\mathrm{CH}_{3}\right)$. Anal. Calcd for $\mathrm{C}_{9} \mathrm{H}_{13} \mathrm{IO}_{3}$ : C, 36.51; $\mathrm{H}, 4.43$. Found: $\mathrm{C}, 36.39 ; \mathrm{H}, 4.35$.

\section{[3a $R, 4 R, 5 S, 6 a S]-4-H y d r o x y m e t h y l-5-m e t h y l-h e x a h y d r o-c y c l o p e n t a[b]$ furan-2-one (7).}

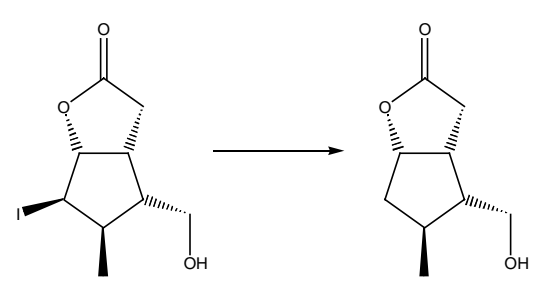

Excess Raney®-nickel [25 $\mathrm{ml}$ of an aqueous suspension (Fluka)] was decanted and the solid washed with $\mathrm{EtOH}(3 \times 1.5 \mathrm{~mL})$. $\mathrm{CH}_{2} \mathrm{Cl}_{2}(3 \mathrm{~mL})$ and three drops of EtOH were then added. Under $\mathrm{N}_{2}$, a solution of lactone $11(110 \mathrm{mg}, 0.37 \mathrm{mmol})$ in $\mathrm{CH}_{2} \mathrm{Cl}_{2}(1$ $\mathrm{mL}$ ) was added, and the mixture was stirred for $1 \mathrm{~h}$ at room temperature, monitoring the reaction by TLC (hexane/EtOAc $=$ 3:7). The mixture was diluted with $\mathrm{CH}_{2} \mathrm{Cl}_{2}$ and filtered. Solvent was evaporated and the residue was separated by column chromatography (hexane/ EtOAc $=1: 1$ ) to yield lactone $7(45 \mathrm{mg}, 0.26 \mathrm{mmol}$, yield $=71 \%) .[\alpha]_{\mathrm{D}}=+14.73\left(c=0.9, \mathrm{CH}_{2} \mathrm{Cl}_{2}\right)$; IR (Nujol) 3450, 2968, 1756, 1306, 1186, 1028, $742 \mathrm{~cm}^{-1} ;{ }^{1} \mathrm{H}$ NMR $\left(300 \mathrm{MHz}, \mathrm{CDCl}_{3}\right) \delta 5.02(\mathrm{t}, J=6 \mathrm{~Hz}$, $1 \mathrm{H}), 3.88(\mathrm{dd}, J=4,10.6 \mathrm{~Hz}, 1 \mathrm{H}), 3.62(\mathrm{dd}, J=8.8,10.6 \mathrm{~Hz}, 1 \mathrm{H}), 3.10-3.22(\mathrm{~m}, 1 \mathrm{H}), 2.64-2.69(\mathrm{~m}$, 2H), 2.23 (dd, $J=5,14 \mathrm{~Hz}, 1 \mathrm{H}), 1.72-1.90(\mathrm{~m}, 2 \mathrm{H}), 1.6$ (brs, OH), 1.40-1.52 (ddd, $J=5.5,11.9$, $17.4 \mathrm{~Hz}, 1 \mathrm{H}), 1.05(\mathrm{~d}, J=6.5 \mathrm{~Hz}, 3 \mathrm{H}) ;{ }^{13} \mathrm{C} \mathrm{NMR}\left(75.2 \mathrm{MHz}, \mathrm{CDCl}_{3}\right) \delta 178.5(\mathrm{C}=\mathrm{O}), 84.9(\mathrm{CH})$, $60.8\left(\mathrm{CH}_{2}\right), 50.4(\mathrm{CH}), 41.4\left(\mathrm{CH}_{2}\right), 40.0(\mathrm{CH}), 32.5(\mathrm{CH}), 29.5\left(\mathrm{CH}_{2}\right), 17.3\left(\mathrm{CH}_{3}\right) ;$ mass $(\%): 170$ $\left(\mathrm{M}^{+}, 10\right), 140$ (85), 95 (47), 81 (100), 69 (35), 55 (40), 41 (41). Anal. Calcd for $\mathrm{C}_{9} \mathrm{H}_{14} \mathrm{O}_{3}$ : C, 63.51; H, 8.29. Found: C, 63.62; H, 8.37.

[3aR,4R,5S,6aS]-5-Methyl-4-(2-trimethylsilanyl-ethoxymethoxymethyl)-hexahydrocyclopenta[b] furan-2-one (12).

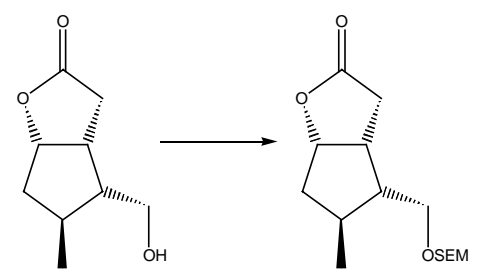

To a solution of lactone 7 (90 $\mathrm{mg}, 0.53 \mathrm{mmol})$ in dry $\mathrm{CH}_{2} \mathrm{Cl}_{2}(2 \mathrm{~mL})$, under Argon, were added, in the order, tetrabutylammonium fluoride (216 mg, $0.58 \mathrm{mmol})$, diisopropylethyl amine $(450 \mu \mathrm{L}, 2.65 \mathrm{mmol})$ and trimethylsilyletoxymethyl chloride $(280 \mu \mathrm{L}, 1.6 \mathrm{mmol})$. The mixture was stirred at room temperature for $3 \mathrm{~h}$ ( TLC: hexane /EtOAc = 3:7). The reaction was diluted with $\mathrm{CH}_{2} \mathrm{Cl}_{2}$ and washed with aq. $\mathrm{NaHSO}_{4}$. The aqueous layer was extracted with $\mathrm{CH}_{2} \mathrm{Cl}_{2}$. The organic layer was dried over $\mathrm{Na}_{2} \mathrm{SO}_{4}$ and solvent was evaporated. Separation of the residue by column chromatography ( $20 \mathrm{mg}$ of silica gel, hexane/EtOAc $=8: 2)$ afforded 12 as colorless oil $(154 \mathrm{mg}$; yield $=97 \%)$. $[\alpha]_{\mathrm{D}}=$ +23.94 ( $c=1.3, \mathrm{CH}_{2} \mathrm{Cl}_{2}$ ); IR (film) 2950, 1770, 1380, 1280, 1250, 1170, 1040, 940, 860, 840, 740 $\mathrm{cm}^{-1} ;{ }^{1} \mathrm{H}$ NMR $\left(300 \mathrm{MHz}, \mathrm{CDCl}_{3}\right) \delta 5.0(\mathrm{t}, J=6.1 \mathrm{~Hz}, 1 \mathrm{H}), 4.66(\mathrm{ABq}, J=6.9 \mathrm{~Hz}, 2 \mathrm{H}), 3.70(\mathrm{dd}, J$ $=10.2,3.8 \mathrm{~Hz}, 1 \mathrm{H}), 3.58-3.68(\mathrm{~m}, 2 \mathrm{H}), 3.42-3.52(\mathrm{~m}, 1 \mathrm{H}), 3.06-3.18(\mathrm{br} q u, J=7 \mathrm{~Hz}, 1 \mathrm{H}), 2.58-$ $2.64(\mathrm{~m}, 2 \mathrm{H}), 2.22(\mathrm{dd}, J=5,13.8 \mathrm{~Hz}, 1 \mathrm{H}), 1.78-1.92(\mathrm{~m}, 2 \mathrm{H}), 1.45(\mathrm{ddd}, J=5.5,11.9,17.4 \mathrm{~Hz}$, $1 \mathrm{H}), 1.04(\mathrm{~d}, J=6.5 \mathrm{~Hz}, 3 \mathrm{H}), 0.93-1.00(\mathrm{~m}, 2 \mathrm{H}), 0.02(\mathrm{~s}, 9 \mathrm{H}, 3 \times \mathrm{Me}) ;{ }^{13} \mathrm{C} \mathrm{NMR}(75.2 \mathrm{MHz}$, $\left.\mathrm{CD}_{2} \mathrm{Cl}_{2}\right) \delta 176.3(\mathrm{CO}), 93.6\left(\mathrm{CH}_{2}\right), 83.3(\mathrm{CH}), 65.1\left(\mathrm{CH}_{2}\right), 63.9\left(\mathrm{CH}_{2}\right), 47.4(\mathrm{CH}), 40.4\left(\mathrm{CH}_{2}\right), 39.3$ 
$(\mathrm{CH}), 31.7(\mathrm{CH}), 28.3\left(\mathrm{CH}_{2}\right), 16.7\left(\mathrm{CH}_{2}\right), 15.9\left(\mathrm{CH}_{3}\right),-3.1\left(3 \mathrm{xCH}_{3}\right)$; ESI: $323.2\left([\mathrm{M}+\mathrm{Na}]^{+}\right), 622.9$ $\left([2 \mathrm{M}+\mathrm{Na}]^{+}\right)$. Anal. Calcd for $\mathrm{C}_{15} \mathrm{H}_{28} \mathrm{O}_{4} \mathrm{Si}: \mathrm{C}, 59.96 ; \mathrm{H}, 9.39$. Found: $\mathrm{C}, 59.83 ; \mathrm{H}, 9.45$.

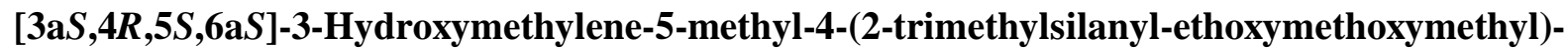
hexahydro-cyclopenta[b]furan-2-one (6).

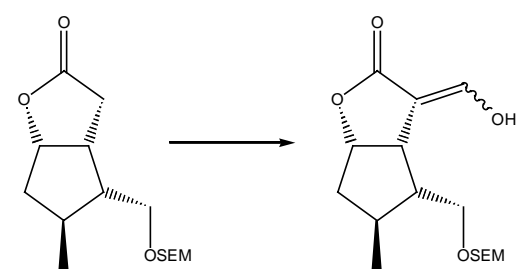

Lactone 12 (150 mg, $0.5 \mathrm{mmol})$ in $\operatorname{dry~} \mathrm{Et}_{2} \mathrm{O}(7 \mathrm{~mL})$ was added to $\mathrm{NaH}(60 \%$ dispersion in oil, $62 \mathrm{mg}, 1.5 \mathrm{mmol})$ in dry $\mathrm{Et}_{2} \mathrm{O}(3 \mathrm{~mL})$ under argon at room temperature. After five minutes, freshly distilled HCOOEt $(600 \mu \mathrm{L}, 7.4 \mathrm{mmol})$ was added and the solution was stirred for $20 \mathrm{~h}$, during which time the color turned to orange. The solution was acidified with $0.6 \mathrm{~N} \mathrm{HCl}$ and the aq layer was extracted with EtOAc. The combined organic layers were dried over $\mathrm{MgSO}_{4}$ and solvent was evaporated under reduced pressure. Separation of the residue by column chromatography [i) hexane; ii) hexane/EtOAc $=1: 1]$ gave enol 6 as colorless oil (158 mg, yield = 96\%); $[\alpha]_{\mathrm{D}}=-37.9\left(c=1.1, \mathrm{CH}_{2} \mathrm{Cl}_{2}\right)$; IR (film) 3600-2600, 2955, 1744, 1648, 1375, 1248, 1194, 1110, 1027, 985, 938, 836, $767 \mathrm{~cm}^{-1} ;{ }^{1} \mathrm{H}$ NMR (300 MHz, $\left.\mathrm{CDCl}_{3}\right) \delta 9.10(\mathrm{~d}, J=13.3 \mathrm{~Hz}$, $1 \mathrm{H}), 7.59(\mathrm{dd}, J=1.9,13.3 \mathrm{~Hz}, 1 \mathrm{H}), 4.94-5.10(\mathrm{~m}, 1 \mathrm{H}), 4.64-4.81(\mathrm{~m}, 2 \mathrm{H}), 3.88(\mathrm{dd}, J=4.3,10.5$ $\mathrm{Hz}, 1 \mathrm{H}) ; 3.60-3.74(\mathrm{~m}, 3 \mathrm{H}), 3.36-3.46(\mathrm{~m}, 1 \mathrm{H}), 2.24(\mathrm{dd}, J=5.2,13.3 \mathrm{~Hz}, 1 \mathrm{H}), 1.80-2.00(\mathrm{~m}, 2 \mathrm{H})$, 1.4-1.5 (m, 1H), $1.02(\mathrm{~d}, J=6.2 \mathrm{~Hz}, 3 \mathrm{H}), 0.94-1.02(\mathrm{~m}, 2 \mathrm{H}), 0.05(\mathrm{~s}, 9 \mathrm{H}, 3 \mathrm{xMe}) ;{ }^{13} \mathrm{C}$ NMR $(75.2$ $\left.\mathrm{MHz}, \mathrm{CDCl}_{3}\right) \delta 193.7(\mathrm{C}), 176.5(\mathrm{C}), 173.2(\mathrm{C}), 159.7(\mathrm{CH}), 156.3(\mathrm{CH}), 102.1(\mathrm{C}), 99.7(\mathrm{C}), 94.9$ $\left(\mathrm{CH}_{2}\right), 94.7\left(\mathrm{CH}_{2}\right), 84.5(\mathrm{CH}), 81.2(\mathrm{CH}), 67.0\left(\mathrm{CH}_{2}\right), 66.5\left(\mathrm{CH}_{2}\right), 66.3\left(\mathrm{CH}_{2}\right), 65.6\left(\mathrm{CH}_{2}\right), 50.6$ $(\mathrm{CH}), 50.3(\mathrm{CH}), 42.8(\mathrm{CH}), 42.7(\mathrm{CH}), 42.1\left(\mathrm{CH}_{2}\right), 41.8\left(\mathrm{CH}_{2}\right), 32.6(\mathrm{CH}), 32.3(\mathrm{CH}), 18.0\left(\mathrm{CH}_{2}\right)$, $17.9\left(\mathrm{CH}_{2}\right), 17.1\left(\mathrm{CH}_{3}\right), 16.8\left(\mathrm{CH}_{3}\right),-1.5\left(3 \mathrm{xCH}_{3}\right),-1.8\left(3 \mathrm{xCH}_{3}\right)$; ESI: $351.2\left([\mathrm{M}+\mathrm{Na}]^{+}\right), 679.0$ $\left([2 \mathrm{M}+\mathrm{Na}]^{+}\right)$. Anal. Calcd for $\mathrm{C}_{16} \mathrm{H}_{28} \mathrm{O}_{5} \mathrm{Si}: \mathrm{C}, 58.50 ; \mathrm{H}, 8.59$. Found: C, 58.62; H, 8.68.

\section{2,3,4,6-tetra- $O$-benzyl- $\alpha$-D-glucopyranosyl bromide (5).}

Oxalyl bromide ( $2 \mathrm{M}$ in $\mathrm{CH}_{2} \mathrm{Cl}_{2}, 3.0 \mathrm{mmol}, 1.6 \mathrm{~mL}$ ) was added to a stirred solution of 2,3,4,6-tetra$O$-benzyl- $\alpha$-D-glucopyranose $(1.3 \mathrm{~g}, 2.3 \mathrm{mmol})$ dissolved in dry $\mathrm{CH}_{2} \mathrm{Cl}_{2}(15 \mathrm{~mL})$ and DMF $(0.84$ $\mathrm{mL}$ ). The mixture was stirred under argon at room temperature for $30 \mathrm{~min}$ and then cooled to $0^{\circ} \mathrm{C}$. The organic layer was washed with cold $\mathrm{H}_{2} \mathrm{O}$ and then dried over $\mathrm{MgSO}_{4}$. Solvent was evaporated and the residual product was immediately used in the following step.

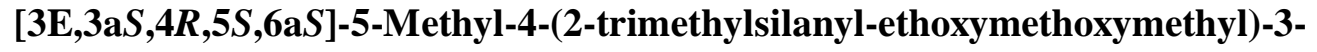
$([2 R, 3 R, 4 S, 5 R, 6 R]-3,4,5$-tris-benzyloxy-6-benzyloxymethyl-tetrahydro-pyran-2yloxymethylene)-hexahydro-cyclopenta[b]furan-2-one (13).
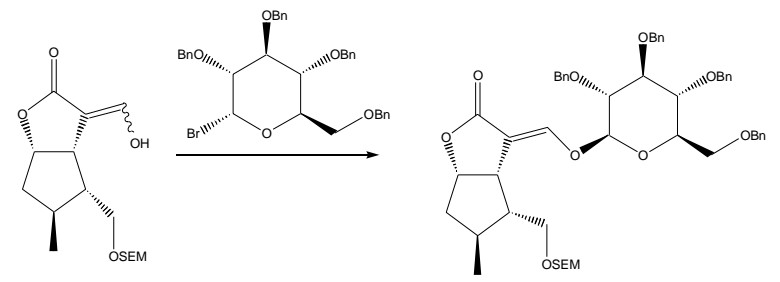

Dry $\mathrm{K}_{2} \mathrm{CO}_{3}$ (250 mg, $\left.1.8 \mathrm{mmol}\right)$ was added to enol 6 (148 mg, $0.45 \mathrm{mmol})$ dissolved in dry NMP $(10 \mathrm{~mL})$ under argon. Five minutes later, 2,3,4,6-tetra- $O$-benzyl- $\alpha$-D-glucopyranosyl bromide (5) (1.4 g, $2.25 \mathrm{mmol})$, dissolved in NMP (3 mL), was added dropwise. The reaction was checked by TLC ( hexane/EtOAc $=7: 3$ ) and went to completion $4 \mathrm{~h}$ later. The mixture was diluted with $\mathrm{Et}_{2} \mathrm{O}$ and washed with $\mathrm{H}_{2} \mathrm{O}$, followed by $5 \%$ aq $\mathrm{NaHCO}_{3}$. The aqueous phase was extracted with EtOAc and the combined organic layers were dried over $\mathrm{MgSO}_{4}$, filtered, and evaporated. Separation of the residue by column chromatography with hexane/ EtOAc $=8: 2$ yielded lactone $13(364 \mathrm{mg}$, yield $=95 \%)$ as colorless solid, m.p. $=86^{\circ} \mathrm{C}-90^{\circ} \mathrm{C} ;[\alpha]_{\mathrm{D}}=-70.3(c=1.1$, $\mathrm{CH}_{2} \mathrm{Cl}_{2}$ ); IR (nujol) 3033, 2952, 1750, 1676, 1478, 1361, 1209, 1071, 835, 736, 697, $678 \mathrm{~cm}^{-1} ;{ }^{1} \mathrm{H}$ NMR $\left(300 \mathrm{MHz}, \mathrm{CDCl}_{3}\right) \delta 7.56(\mathrm{~d}, J=1.9 \mathrm{~Hz}, 1 \mathrm{H}), 7.12-7.48(\mathrm{~m}, 20 \mathrm{H}), 4.74-5.00(\mathrm{~m}, 7 \mathrm{H}), 4.46-$ $4.66(\mathrm{~m}, 5 \mathrm{H}), 3.45-3.85(\mathrm{~m}, 11 \mathrm{H}), 2.24(\mathrm{dd}, J=4.8,13.6 \mathrm{~Hz}, 1 \mathrm{H}), 1.80-2.00(\mathrm{~m}, 2 \mathrm{H}), 1.43$ (ddd, $J=$ 
5, 11.2, $16.2 \mathrm{~Hz}, 1 \mathrm{H}), 1.08(\mathrm{~d}, J=6.0 \mathrm{~Hz}, 3 \mathrm{H}), 0.88-0.96(\mathrm{~m}, 2 \mathrm{H}), 0.05(\mathrm{~s}, 9 \mathrm{H}, 3 \mathrm{xMe}) ;{ }^{13} \mathrm{C} \mathrm{NMR}$ $\left(75.2 \mathrm{MHz}, \mathrm{CD}_{2} \mathrm{Cl}_{2}\right) \delta 173.1(\mathrm{CO}), 153.6(\mathrm{CH}), 139.4(\mathrm{C}), 139.0(\mathrm{C}), 129.0(\mathrm{CH}), 128.7(\mathrm{CH})$, $128.6(\mathrm{CH}), 128.57(\mathrm{CH}), 128.53(\mathrm{CH}), 128.33(\mathrm{CH}), 109.3(\mathrm{C}), 105.1\left(\right.$ anomeric $\mathrm{CH}, J_{\mathrm{CH}}^{1}=162$ $\mathrm{Hz}), 95.8\left(\mathrm{CH}_{2}\right), 84.9(\mathrm{CH}), 83.8(\mathrm{CH}), 82.3(\mathrm{CH}), 78.0(\mathrm{CH}), 76.4(\mathrm{CH}), 76.3\left(\mathrm{CH}_{2}\right), 75.7(\mathrm{CH})$, $74.2\left(2 \mathrm{xCH}_{2}\right), 69.2\left(\mathrm{CH}_{2}\right), 67.3\left(\mathrm{CH}_{2}\right), 65.5\left(\mathrm{CH}_{2}\right), 51.5\left(\mathrm{CH}_{2}\right), 44.4(\mathrm{CH}), 42.2(\mathrm{CH}), 34.8\left(\mathrm{CH}_{2}\right)$, $(\mathrm{CH}), 18.9\left(\mathrm{CH}_{2}\right), 18.3\left(\mathrm{CH}_{3}\right),-0.9\left(3 \mathrm{xCH}_{3}\right)$; ESI: $873.5\left([\mathrm{M}+\mathrm{Na}]^{+}\right), 1723.2\left([2 \mathrm{M}+\mathrm{Na}]^{+}\right)$. Anal. Calcd for $\mathrm{C}_{50} \mathrm{H}_{62} \mathrm{O}_{10} \mathrm{Si}$ : C, 70.56; H, 7.34. Found: C, 70.67; H, 7.42.

[3E,3a $S, 4 R, 5 S, 6 \mathrm{a} S]-4-H y d r o x y m e t h y l-5-m e t h y l-3-([2 R, 3 R, 4 S, 5 R, 6 R]-3,4,5-$ tris-benzyloxy-6benzyloxymethyl-tetrahydro-pyran-2-yloxymethylene)-hexahydro-cyclopenta[b]furan-2-one (4).
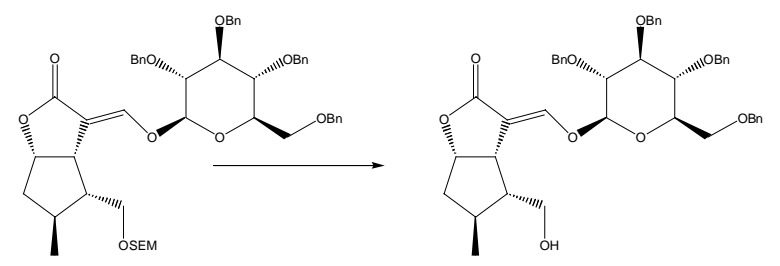

$\mathrm{nBuSH}(30 \mu \mathrm{L}, 0.225 \mathrm{mmol})$ and $\mathrm{MgBr}_{2}(56 \mathrm{mg}$, $0.27 \mathrm{mmol}$ ) were added in the order to lactone $\mathbf{1 3}$ $(80 \mathrm{mg}, 0.09 \mathrm{mmol})$ dissolved in $\operatorname{dry~}^{\mathrm{Et}} \mathrm{t}_{2} \mathrm{O}(1.5$ $\mathrm{mL})$ and $\mathrm{CH}_{2} \mathrm{Cl}_{2}(1.5 \mathrm{~mL})$. The mixture was stirred for $7 \mathrm{~h}$ at $\mathrm{rt}$, monitored by TLC (hexane/EtOAc $=6: 4$ ), diluted with $\mathrm{CH}_{2} \mathrm{Cl}_{2}$, and washed with $5 \%$ aq $\mathrm{NaHCO}_{3}$. The aqueous layer was extracted with $\mathrm{CH}_{2} \mathrm{Cl}_{2}$. The combined organic layers were dried over $\mathrm{Na}_{2} \mathrm{SO}_{4}$, filtered, and evaporated. The residue was separated by column chromatography. Elution with hexane/EtOAc $=75: 25$ afforded free alcohol 4 (48 mg, yield $=75 \%)$ as a pale yellow oil; $[\alpha]_{\mathrm{D}}=-57.8\left(c=0.495, \mathrm{CH}_{2} \mathrm{Cl}_{2}\right)$; IR (film) $3583,3031,2872,1738,1676$, $1454,1372,1308,1242,1070,847,738,699 \mathrm{~cm}^{-1} ;{ }^{1} \mathrm{H}$ NMR $\left(300 \mathrm{MHz}, \mathrm{CDCl}_{3}\right) \delta 7.57(\mathrm{~d}, J=2.1$ $\mathrm{Hz}, 1 \mathrm{H}), 7.15-7.40(\mathrm{~m}, 20 \mathrm{H}), 4.74-4.96(\mathrm{~m}, 7 \mathrm{H}), 4.48-4.66(\mathrm{~m}, 3 \mathrm{H}), 3.44-3.80(\mathrm{~m}, 9 \mathrm{H}), 2.68(\mathrm{dd}, J$ $=8.0,6.6 \mathrm{~Hz}, 1 \mathrm{H}), 2.23(\mathrm{dd}, J=5.4,13.8 \mathrm{~Hz}, 1 \mathrm{H}), 1.76-1.92(\mathrm{~m}, 1 \mathrm{H}), 1.35-1.65(\mathrm{~m}, 2 \mathrm{H}), 1.04(\mathrm{~d}, J$ $=6.2 \mathrm{~Hz}, 3 \mathrm{H}) ;{ }^{13} \mathrm{C}$ NMR $\left.\left(75.2 \mathrm{MHz}, \mathrm{CD}_{2} \mathrm{Cl}_{2}\right) \delta 172.7(\mathrm{CO}), 153.7 \mathrm{CH}\right), 139.3(\mathrm{C}), 138.98(\mathrm{C})$, $138.93(\mathrm{C}), 138.8(\mathrm{C}), 129.1(\mathrm{CH}), 129.0(\mathrm{CH}), 128.74(\mathrm{CH}), 128.70(\mathrm{CH}), 128.6(\mathrm{CH}), 128.5$ $(\mathrm{CH}), 128.4(\mathrm{CH}), 128.35(\mathrm{CH}), 109.6(\mathrm{C}), 105.3(\mathrm{CH}), 84.8(\mathrm{CH}), 83.7(\mathrm{CH}), 82.1(\mathrm{CH}), 77.8$ $(\mathrm{CH}), 76.6(\mathrm{CH}), 76.2\left(\mathrm{CH}_{2}\right), 75.8\left(\mathrm{CH}_{2}\right), 75.7\left(\mathrm{CH}_{2}\right), 74.2\left(\mathrm{CH}_{2}\right), 69.0\left(\mathrm{CH}_{2}\right), 61.7\left(\mathrm{CH}_{2}\right), 54.8$ $(\mathrm{CH}), 43.9(\mathrm{CH}), 42.3\left(\mathrm{CH}_{2}\right), 33.3(\mathrm{CH}), 17.9\left(\mathrm{CH}_{3}\right)$; ESI: $743.5\left([\mathrm{M}+\mathrm{Na}]^{+}\right)$. Anal. Calcd for $\mathrm{C}_{44} \mathrm{H}_{48} \mathrm{O}_{9}$ : C, 73.31; H, 6.71. Found: C, 73.12; H, 6.81.

$[2 \mathrm{a} S, 4 S, 4 \mathrm{a} R, 7 R, 7 \mathrm{a} S, 7 \mathrm{~b} S]-4-M e t h y l-7-([2 S, 3 R, 4 S, 5 R, 6 R]-3,4,5-$ tris-benzyloxy-6benzyloxymethyl-tetrahydro-pyran-2-yloxy)-octahydro-2,6-dioxa-cyclopenta[ $[c d]$ inden-1-one (15).

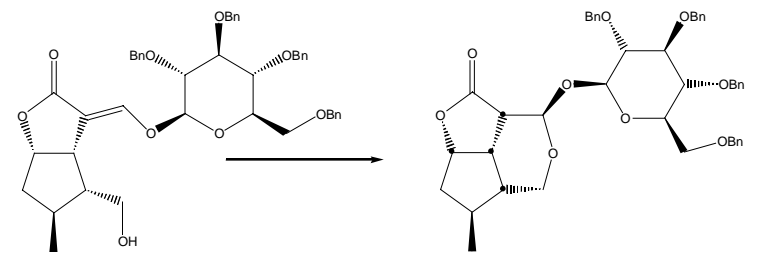

$\mathrm{Hg}\left(\mathrm{OCOCF}_{3}\right)_{2}(136 \mathrm{mg}, 0.32 \mathrm{mmol})$ was added to alcohol 4 (58 $\mathrm{mg}, 0.08 \mathrm{mmol})$ dissolved in dry THF $(2 \mathrm{~mL})$ under argon, and the mixture was stirred at $\mathrm{rt}$ for $4 \mathrm{~d}$, monitored by TLC (hexane/EtOAc $=6: 4)$. Solvent was evaporated and the crude product was dissolved in degassed THF/

$5 \%$ aq $\mathrm{NaOH}$, giving an orange solution. $\mathrm{NaBH}_{4}$ was added until complete discoloration, followed by acetone to destroy excess $\mathrm{NaBH}_{4}$. Mercury was separated and the supernatant solution was diluted with EtOAc and brine. The aq layer was extracted with EtOAc and the combined organic layers were washed with brine, dried over $\mathrm{Na}_{2} \mathrm{SO}_{4}$, and evaporated. Separation of the residue by column chromatography (hexane/EtOAc $=8: 2)$ yielded lactone 15 as colorless solid $(30 \mathrm{mg}$, yield = $50 \%)$, m.p. $=125^{\circ} \mathrm{C}-130^{\circ} \mathrm{C} ;[\alpha]_{\mathrm{D}}=+50.2\left(c=0.8, \mathrm{CH}_{2} \mathrm{Cl}_{2}\right)$; IR (nujol) 3033, 2918, 1763, 1453, 1354, 1073, 1000, 909, $732 \mathrm{~cm}^{-1} ;{ }^{1} \mathrm{H}$ NMR (400 MHz, $\left.\mathrm{CDCl}_{3}\right) \delta 7.1-7.4(\mathrm{~m}, 20 \mathrm{H}), 5.52(\mathrm{~s}, 1 \mathrm{H}, 3-$ $\mathrm{H})$, 4.5-5.0 (m, 10H), $4.35(\mathrm{dd}, J=4,13 \mathrm{~Hz}, 1 \mathrm{H}, 1 \beta-\mathrm{H}), 3.6-3.8(\mathrm{~m}, 4 \mathrm{H}), 3.45-3.6(\mathrm{~m}, 3 \mathrm{H}), 3.15$ 
(ddd, $J=6.6,10.3,13 \mathrm{~Hz}, 1 \mathrm{H}, 5-\mathrm{H}), 2.63(\mathrm{~d}, J=10.3 \mathrm{~Hz}, 1 \mathrm{H}, 4-\mathrm{H}), 2.25(\mathrm{dd}, J=6.7,13.4 \mathrm{~Hz}, 1 \mathrm{H}$, $7 \alpha-\mathrm{H}), 1.95-2.10(\mathrm{~m}, 1 \mathrm{H}, 8-\mathrm{H}), 1.25-1.40(\mathrm{~m}, 2 \mathrm{H}, 7 \beta-\mathrm{H}$ and $9-\mathrm{H}), 1.05\left(\mathrm{~d}, J=6.4 \mathrm{~Hz}, 3 \mathrm{H}, \mathrm{CH}_{3}\right)$; ${ }^{13} \mathrm{C}$ NMR (75.2 MHz, $\left.\mathrm{CD}_{2} \mathrm{Cl}_{2}\right) \delta 175.8(\mathrm{CO}), 139.6(\mathrm{C}), 139.3(\mathrm{C}), 139.2(\mathrm{C}), 139.1(\mathrm{C}), 129.51$ $(\mathrm{CH}), 129.13(\mathrm{CH}), 129.04(\mathrm{CH}), 129.02(\mathrm{CH}), 128.82(\mathrm{CH}), 128.63(\mathrm{CH}), 128.61(\mathrm{CH}), 128.50$ $(\mathrm{CH}), 128.43(\mathrm{CH}), 128.38(\mathrm{CH}), 128.32(\mathrm{CH}), 128.26(\mathrm{CH}), 103.1(\mathrm{CH}), 98.2(\mathrm{CH}), 85.5(\mathrm{CH})$, $84.5(\mathrm{CH}), 83.2(\mathrm{CH}), 78.4(\mathrm{CH}), 76.2\left(\mathrm{CH}_{2}\right), 75.8\left(\mathrm{CH}_{2}\right), 75.7(\mathrm{CH}), 75.6\left(\mathrm{CH}_{2}\right), 74.0\left(\mathrm{CH}_{2}\right), 69.6$ $\left(\mathrm{CH}_{2}\right), 57.0\left(\mathrm{CH}_{2}\right), 42.2\left(\mathrm{CH}_{2}\right), 42.1(\mathrm{CH}), 42.0(\mathrm{CH}), 36.6(\mathrm{CH}), 33.3(\mathrm{CH}), 17.1\left(\mathrm{CH}_{3}\right)$; ESI: 743.5 $\left([\mathrm{M}+\mathrm{Na}]^{+}\right)$. Anal. Calcd for $\mathrm{C}_{44} \mathrm{H}_{48} \mathrm{O}_{9}$ : C, 73.31; H, 6.71. Found: C, 73.42; H, 6.81.

[2aS $, 4 S, 4 \mathrm{a} R, 7 R, 7 \mathrm{a} S, 7 \mathrm{bS}]-4-M e t h y l-7-([2 S, 3 R, 4 R, 5 R, 6 R]-3,4,5$-trihydroxy-6-hydroxymethyltetrahydro-pyran-2-yloxy)-octahydro-2,6-dioxa-cyclopenta[cd]inden-1-one (1). (Semperoside A)
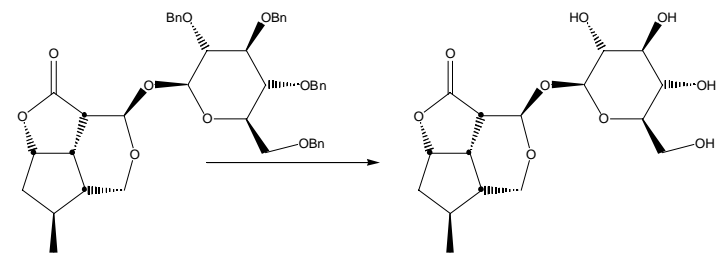

A catalytic amount of palladium, 5 wt. \% on activated carbon, was added to lactone $\mathbf{1 5}(21 \mathrm{mg}$, $0.029 \mathrm{mmol})$ dissolved in EtOAc $(2 \mathrm{~mL})$ and $\mathrm{MeOH}$ $(1 \mathrm{~mL})$. The mixture was stirred under hydrogen at $\mathrm{rt}$ for $30 \mathrm{~min}$, monitoring the reaction by TLC (EtOAc / $\mathrm{EtOH} /$ toluene $=4: 2: 1$ ). The mixture was filtered and solvent was evaporated. Separation of the residue by column chromatography (EtOAc / EtOH / toluene $=4: 1: 1)$ afforded compound 1 (semperoside A) $(10 \mathrm{mg}$, yield $=95 \%$. $)$ as colorless solid, $\mathrm{mp}$ $182-184{ }^{\circ} \mathrm{C} ;[\alpha]_{\mathrm{D}}=+67.2(c=0.3, \mathrm{MeOH}) ; \mathrm{CD}=215 \mathrm{~nm}\left(\Delta \varepsilon=-1.3 ; c=10^{-3} \mathrm{M}\right.$ in $\left.\mathrm{MeOH}\right) ;{ }^{1} \mathrm{H}$ NMR (400 MHz, D $2 \mathrm{O}) \delta 5.3(\mathrm{~s}, 1 \mathrm{H}, 3-\mathrm{H}), 5.05(\mathrm{dd}, J=4.5,5.5 \mathrm{~Hz}, 1-\mathrm{H}, 6-\mathrm{H}), 4.6(\mathrm{~d}, J=8 \mathrm{~Hz}, 1 \mathrm{H}$, 1'-H), 4.18 (dd, $J=4.1,12.7 \mathrm{~Hz}, 1 \mathrm{H}, 1 \beta-\mathrm{H}), 3.79$ (dd, $J=3,11.4 \mathrm{~Hz}, 1 \mathrm{H}, 6$ ' -H), 3.65 (dd, $J=4.6$, $\left.11.4 \mathrm{~Hz}, 1 \mathrm{H}, 6^{\prime}-\mathrm{H}^{\prime}\right), 3.57$ (br d, $\left.J=12.7 \mathrm{~Hz}, 1 \mathrm{H}, 1 \alpha-\mathrm{H}\right), 3.2-3.5(\mathrm{~m}, 5 \mathrm{H}), 3.06(\mathrm{~d}, J=10.5 \mathrm{~Hz}, 1 \mathrm{H}$, 4-H), 2.09 (dd, $J=5.3,14.2 \mathrm{~Hz}, 1 \mathrm{H}, 7 \alpha-\mathrm{H}), 1.65-1.78$ (m, 1H, 8-H), 1.43 (ddd, $J=4,10,12 \mathrm{~Hz}$, $1 \mathrm{H}, 9-\mathrm{H}), 1.35(\mathrm{ddd}, J=4.3,13,14 \mathrm{~Hz}, 1 \mathrm{H}, 7 \beta-\mathrm{H}), 0.92\left(\mathrm{~d}, J=6.4 \mathrm{~Hz}, 3 \mathrm{H}, \mathrm{CH}_{3}\right) ;{ }^{13} \mathrm{C} \mathrm{NMR}(75.2$ $\left.\mathrm{MHz}, \mathrm{D}_{2} \mathrm{O}\right) \delta 178.9(\mathrm{CO}), 102.5(\mathrm{CH}), 97.5(\mathrm{CH}), 86.7(\mathrm{CH}), 76.6(\mathrm{CH}), 75.9(\mathrm{CH}), 73.6(\mathrm{CH})$, $69.7(\mathrm{CH}), 60.8\left(\mathrm{CH}_{2}\right), 57.2\left(\mathrm{CH}_{2}\right), 41.7(\mathrm{CH}), 40.8\left(\mathrm{CH}_{2}\right), 40.7(\mathrm{CH}), 35.6(\mathrm{CH}), 32.8(\mathrm{CH}), 15.9$ $\left(\mathrm{CH}_{3}\right)$; ESI: $383.2\left([\mathrm{M}+\mathrm{Na}]^{+}\right), 743.1\left([2 \mathrm{M}+\mathrm{Na}]^{+}\right)$;. Anal. Calcd for $\mathrm{C}_{16} \mathrm{H}_{24} \mathrm{O}_{9}: \mathrm{C}, 53.33 ; \mathrm{H}, 6.71$. Found: C, 53.21; H, 6.63 .

Iridoid atomic numbering in NMR-spectra:
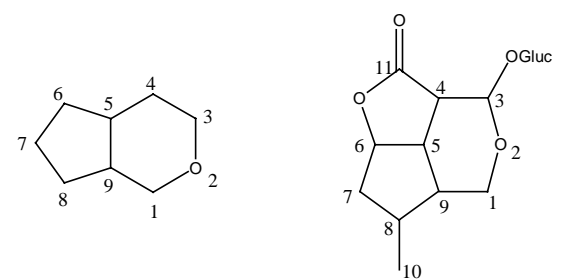